\title{
Lentivirus-mediated short-hairpin RNA targeting IGF-1R inhibits growth and lymphangiogenesis in breast cancer
}

\author{
YANING CHEN $^{1}, \mathrm{CHENFANG} \mathrm{ZHU}^{1}, \mathrm{ZHIYOU} \mathrm{PENG}^{1}$, YALEI DAI ${ }^{2}$ and YAN GU $^{1}$ \\ ${ }^{1}$ Department of General Surgery, Shanghai Ninth People's Hospital, Shanghai Jiaotong University \\ School of Medicine, HuangPu District, Shanghai 200011; ${ }^{2}$ Department of Immunology, Shanghai \\ Tongji University School of Medicine, YangPu District, Shanghai 200092, P.R. China
}

Received April 15, 2012; Accepted July 17, 2012

DOI: $10.3892 /$ or.2012.1964

\begin{abstract}
In this study, we investigated the effects of lentivirus (LV)-mediated short hairpin RNA (shRNA) targeting IGF-1R on the growth and lymphangiogenesis of breast cancer. The LV vector effectively delivered the IGF-1R shRNA to MDA-MB-231 cells, leading to significant reduction of IGF-1R mRNA and protein expression. Infection of MDA-MB-231 cells with LV-IGF-1R shRNA reduced cell growth and migration. Transplantation of MDA-MB-231 cells with suppressed IGF-1R expression in SCID mice reduced tumor growth and lymphangiogenesis. These data collectively suggest that LV-mediated shRNA is an effective way to suppress IGF-1R expression and to inhibit growth and lymphangiogenesis of breast cancer. Specific inhibition of IGF-1R expression with shRNA represents a promising approach for the treatment of breast cancer.
\end{abstract}

\section{Introduction}

Measured by incidence, mortality and economic costs, the global burden of breast cancer in women is substantial and on the increase (1). It is estimated that every year, more than one million women are diagnosed with breast cancer worldwide, and more than 410,000 will die from the disease, representing $14 \%$ of female cancer deaths (2-4). The mortality from breast cancer is mainly due to metastatic disease. While both tamoxifen and trastuzumab are very successful in treating some breast cancer patients, others do not benefit from these therapies, developing resistance to estrogen manipulation, and suffer from progressive metastatic disease (5). The lymphatic system constitutes one of the most important pathways of

Correspondence to: Dr Yan Gu, Department of General Surgery, Shanghai Ninth People's Hospital, Shanghai Jiaotong University School of Medicine, Zhizaoju Road 639, HuangPu District, Shanghai 200011, P.R. China

E-mail: yangu@sjtu.edu.cn

Key words: tumor growth, breast neoplasm, lymphatic metastasis, RNA interference, targeted therapy tumor dissemination. Studies of tumor models in animals and clinicopathological data have indicated that growth of lymphatic vessels (lymphangiogenesis) in the vicinity of solid tumors may contribute to lymphatic metastasis. The most extensively studied signaling system that promotes lymphangiogenesis involves the secreted lymphangiogenic proteins vascular endothelial growth factor-C (VEGF-C) and VEGF-D, and their cognate receptor VEGF receptor-3 (VEGFR-3) (6).

Insulin-like growth factor-1 receptor (IGF-1R) is a glycosylated heterotetramer composed of 2 extracellular $\alpha$ subunits and $\beta$ subunits that have intrinsic tyrosine kinase activity with $70 \%$ homology to the insulin receptor (7). IGF-1R mainly mediates the effect of insulin-like growth factors (IGFs), which are potent mitogens that regulate cell proliferation, differentiation, and protection from apoptosis (8). IGF-1R expression level in primary breast cancer was observed upregulated in $43.8 \%$ of tumors detected by immunohistochemical analysis (9). Among node-negative patients, those with high levels of IGF-1R were found to have significantly reduced overall survival (10). The IGF-IR pathway plays an important role in mediating resistance to both general cytotoxic therapies, such as radiation and chemotherapy, and targeted therapies, such as tamoxifen and trastuzumab (11). Therefore, targeting the IGF pathway might be a novel approach to overcoming this resistance and improving clinical outcome of breast cancer.

RNA interference (RNAi), a novel strategy of gene silencing, has rapidly become a powerful tool for drug discovery and target validation in cell culture (12). The natural role of RNAi is thought to be a cellular defense against viral infection or potentially harmful destabilizing genomic intruders such as transposons. RNAi can also be induced in mammalian cells by the introduction of synthetic small interfering RNA (siRNA) 21-23 base pairs in length, or by plasmid and viral vector systems that express short hairpin RNAs (shRNA) that are subsequently processed to siRNA by the cellular machinery (13-15).

To investigate the potential value of targeting IGF-1R in breast cancer, we utilised the lentivirus-based shRNA expression plasmid pLL3.7 to knock down expression of endogenous IGF-1R. We show that lentivirus (LV)-mediated shRNA that targets IGF-1R can effectively inhibit the growth and migration of MDA-MB-231 breast cancer cells both in vitro and in vivo. 


\section{Materials and methods}

Cell culture. The MDA-MB-231 breast cancer and the human embryonic kidney 293T cell lines were purchased from Nanjing KeyGen Biotech Co., Ltd. (Nanjing, China). MDA-MB-231 cells and 293T cells were cultured in RPMI-1640 (Gibco, Grand Island, NY, USA) and Dulbecco's modified Eagle's medium (DMEM; Gibco), respectively, and supplemented with $10 \%$ fetal bovine serum (FBS; Gibco). Cells were maintained in a humidified incubator with $5 \% \mathrm{CO}_{2}$ at $37^{\circ} \mathrm{C}$.

shRNA design and vector construction. The shRNA expression cassette contained 21 nucleotide (nt) of the target sequence followed by the loop sequence (TTCAAGAGA), reverse complement to the $21 \mathrm{nt}$, stop codon for U6 promoter and Xho1 site (sense strand: 5'-TGAGACCTGAAAGGAAGCGGAGA TTCAAGAGATCTCCGCTTCCTTTCAGGTCTCTTTTT TC-3'; antisense strand: 5'-TCGAGAAAAAAGAGACCTGA AAGGAAGCGGAGATCTCTTGAATCTCCGCTTCCTTT CAGGTCTCA-3'). The IGF-1R-shRNA contains the sense targeting sequence of AGACCTGAAAGGAAGCGGAGA corresponding to the 2250-2270 nucleotide positions of human IGF-1R coding sequence (GenBank accession NM_000875.3). The shRNA cassettes and their complementary strands were synthesized by Shanghai Sangon Biotech Co., and annealed by heating to $95^{\circ} \mathrm{C}$ for $5 \mathrm{~min}$ followed by cooling to room temperature. The resulting double-strand oligo DNA was cloned into the lentivirus-based shRNA expression plasmid pLL3.7 (available from Shanghai Telebio Biomedical Co., Ltd., Shanghai, China), and inserted between $\mathrm{HpaI}$ and $\mathrm{XhoI}$ sites (16). Plasmid pLL3.7 without any shRNA inserted was used as negative control. The resulting plasmid was confirmed by restriction enzyme digestion and DNA sequencing. Plasmid pLL3.7 is designed to contain an EGFP reporter gene controlled by the CMV promoter, enabling the monitoring of lentivirus infection through EGFP expression (17).

Lentiviral vector production. A four-plasmid transfection system (available from Shanghai Telebio Biomedical Co.) was used to produce high-titer lentiviral vectors. Briefly, the packing plasmids and pLL3.7 were amplified in Escherichia coli and purified using AxyPrep Plasmid Maxiprep kit according to the manufacturer's instructions. The lentiviral vectors were then prepared by transfecting $293 \mathrm{~T}$ cells with the plasmids pLL3.7, using calcium phosphate transfection method in the presence of the packaging plasmid pMD2G, pMDLg/pRRE and pRSVrev. The viral supernatant was collected at $48 \mathrm{~h}$ after transfection, concentrated, and passed through a $0.45 \mu \mathrm{m}$ filter. Titers were determined by infecting HeLa cells with serial dilutions of concentrated lentivirus. For a typical preparation, the titre was $\sim 1 \times 10^{7}$ infectious units (IU) per $\mathrm{ml}$. The lentiviral stocks were stored in small aliquot at $-70^{\circ} \mathrm{C}$ for future use.

Cell infection. MDA-MB-231 cells were seeded in 6-well plates $\left(5 \times 10^{5} /\right.$ well $)$ and were cultured overnight. Lentiviruses $(0.1 \mathrm{ml})$ were mixed with $1.5 \mathrm{ml}$ complete medium and added to the cells for incubation for $24 \mathrm{~h}$ at $37^{\circ} \mathrm{C}$. After 24 -h infection, the medium was replaced with fresh 1640 medium. This procedure was repeated for 3 days. The efficiency of transduction was assessed and photomicrographs of EGFP expression were recorded using a Nikon Eclipse TE2000U inverted microscope equipped with a charge-coupled device (CCD) camera.

Real-time polymerase chain reaction (qPCR). Total RNA was isolated using TRIzol reagent (ShineGene Molecular Biotech Co., Shanghai, China) according to the manufacturer's instructions. From total RNA, $1 \mu \mathrm{g}$ was reverse-transcribed into cDNA with EnergicScript First Strand cDNA Synthesis kits (ShineGene Molecular Biotech Co.). Human $\beta$-actin RNA was used as an internal control. Primers for IGF-1R were: forward, 5'-ACAAGTTGAGGATCAGCGAGAATG-3'; reverse, 5'-GG ACAGCGACGGGCAGAG-3'. Gene expression levels were evaluated by real-time quantitative PCR kinetics with ShineSybr Real-Time qPCR MasterMix kits (ShineGene Molecular Biotech Co.). Real-time PCR was performed with $2 \mu \mathrm{l}$ of appropriate diluted cDNA, $1 \mu \mathrm{l}(25 \mathrm{pmol} / \mu \mathrm{l})$ of forward and reverse primers specific for human IGF-1R and $\beta$-actin, $25 \mu \mathrm{l}$ of Hotstart fluo-PCR mix, and $21 \mu 1$ of $\mathrm{ddH}_{2} \mathrm{O}$. Real-time PCR was carried out using the iQ5 real-time PCR detection system (Bio-Rad Laboratories, Hercules, CA, USA) with the following program: pre-heating $94^{\circ} \mathrm{C} 4 \mathrm{~min}$; then 40 cycles of $94 \mathrm{C} 30 \mathrm{sec} ; 60^{\circ} \mathrm{C} 30 \mathrm{sec}$; and $72^{\circ} \mathrm{C} 30 \mathrm{sec}$. The expression of target RNA relative to $\beta$ actin was calculated based on the threshold cycle $\left(\mathrm{C}_{\mathrm{t}}\right)$ as $\mathrm{R}=2^{-\Delta(\Delta \mathrm{C})}$, where $\Delta \mathrm{C}_{\mathrm{t}}=\mathrm{C}_{\mathrm{tIGF}-1 \mathrm{R}}-\mathrm{C}_{\mathrm{tbeta}}$ actin ; $\Delta\left(\Delta \mathrm{C}_{\mathrm{t}}\right)=\Delta \mathrm{Ct}_{\text {sample }}-\Delta \mathrm{C}_{\mathrm{t} \text { control }}$.

Western blotting. Cell lysates were prepared in RIPA buffer (Cell Signaling Technology, Beverly, MA, USA); their protein concentrations were determined using the BCA Protein Assay kit (KeyGen Biotech). For electrophoresis, $30 \mu \mathrm{g}$ of total protein in 5X loading buffer was loaded to each well of a $10 \%$ (w/v) SDS-PAGE gel and transferred to polyvinylidene fluoride (PVDF) membranes. After electrotransferring, the blot was blocked and probed with primary antibody at $4^{\circ} \mathrm{C}$ followed by incubation with horseradish peroxidase (HRP)-conjugated secondary antibody. IGF-I receptor- $\beta$ antibody (Cell Signaling Technology, Beverly, MA, USA) was used at 1/1000 dilution, while a mouse monoclonal antibody against human GAPDH (Abmart, Shanghai, China) at 1:5000 was used as control. Immunoblots were developed using BeyoECL Plus (Beyotime Institute of Biotechnology, Jiangsu Province, China) according to the manufacturer's instructions.

Cell proliferation assay. Cell proliferation was determined by WST-8 assay using Cell Counting Kit-8 (CCK-8; Beyotime Institute of Biotechnology). MDA-MB-231 cells infected or uninfected with LVs were trypsinized counted and seeded in a 96-well plate $\left(3 \times 10^{4} / \mathrm{ml}\right)$ for overnight incubation. Then cells were inoculated with $10 \mu \mathrm{l} \mathrm{CCK}-8$ solution at $37^{\circ} \mathrm{C}$ in a humid atmosphere containing $5 \% \mathrm{CO}_{2}$ for $2 \mathrm{~h}$, and absorbance at $450 \mathrm{~nm}$ of the supernatant was measured spectrophotometrically. This assay was carried out at various time points (at 24, 48 and $72 \mathrm{~h}$ after seeding). A total of three independent experiments were performed, and the means were used to depict the growth curve.

Migration assay. A Transwell system (Corning, NY, USA) was used to evaluate cell migration. The upper and lower chambers were separated by a polycarbonate membrane with pores of 
A

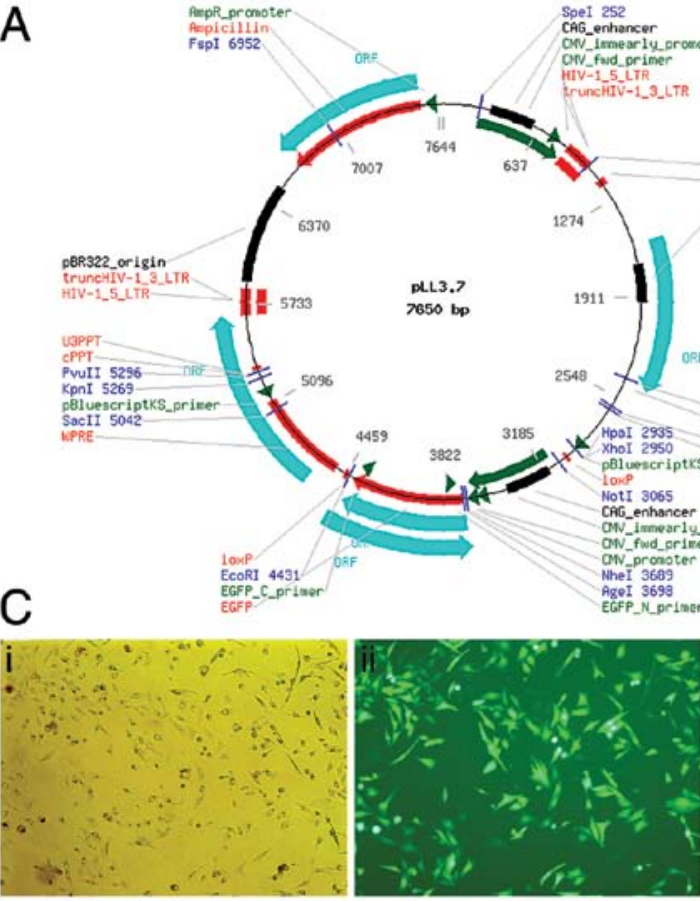

B

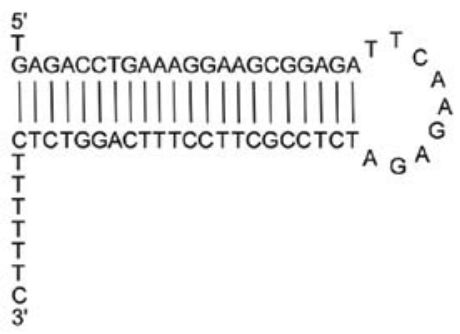

Figure 1. Lentiviruses effectively infect MDA-MB-231 cells. (A) Schematic diagram of plasmid pLL3.7 (17). (B) The stem loop shows a representative hairpin structure of transcribed IGF-1R shRNA. (C) Representative bright field (i and iii) and fluorescent (ii and iv) photomicrographs, showing the transduction efficiency of both LV-IGF-1R shRNA (i and ii) and LV-con (iii and iv) as evident by EGFP expression (ii and iv) (x100 magnification).

$8 \mu \mathrm{m}$, which was coated with fibronectin (BD Biosciences, San Jose, CA, USA) on the lower surface. Approximately $5 \times 10^{3}$ cells suspended in $100 \mu \mathrm{l}$ serum-free medium were seeded onto the upper chamber, and $500 \mu \mathrm{l}$ of culture medium with $10 \%$ FBS was added to the lower chamber. After $24 \mathrm{~h}$ of incubation at $37^{\circ} \mathrm{C}$ with $5 \% \mathrm{CO}_{2}$, the medium was removed from the upper chamber. The non-invaded cells on the upper side of the chamber were scraped off with a cotton swab. Cells on the underside of the membrane were fixed, stained with crystal violet and mounted. The migration activity of cancer cells was determined by counting the cells under a microscope, in 4 different viewing fields, at x200 magnification. Each assay was repeated three times.

Animal experiments. Four-week-old female severe combined immunodeficient (SCID) mice were purchased from Slac Laboratory Animal Co., Ltd., (Shanghai, China), and maintained in the specific pathogen-free (SPF) facility. All animal protocols used for this study were approved by the Institutional Animal Care and Use Committee. MDA-MB-231 cells were infected with LVs as described above and harvested. The infected cells were washed with PBS, counted and resuspended in PBS at $1 \times 10^{7} / \mathrm{ml}$. Female SCID mice (6 mice/group) were injected with $100 \mu \mathrm{l}$ cell suspension subcutaneously to the left inguinal mammary fat pads.The mice were sacrificed on Day 60, and the tumors were measured and removed for immunohistochemical analysis. The tumor volume was calculated as length $(\mathrm{mm}) \times$ the square of the width $\left(\mathrm{mm}^{2}\right) \times \pi / 6$.

Immunohistochemistry. Immunohistochemical (IHC) staining was performed using the Dako EnVision system. Briefly, serial $5-\mu \mathrm{m}$-thick sections were cut from formalin-fixed and paraffin-embedded tumor blocks, dewaxed in xylene, rehydrated through sequential changes of alcohol, and then antigen retrieved in $0.01 \mathrm{M}$ citrate buffer, $\mathrm{pH} 6.0$, at $90^{\circ} \mathrm{C}$ for $20 \mathrm{~min}$. After washing with phosphate-buffered saline (PBS), the tissue sections were incubated with fresh $3 \%$ hydrogen peroxide for $20 \mathrm{~min}$ at room temperature. Sections were blocked with $20 \%$ goat serum for 30 min and incubated with IGF-1R primary antibody (1:50 dilution; Abcam, Cambridge, UK), or lymphatic vessel endothelial receptor 1 (LYVE-1) antibody (1:200 dilution; Abcam) for $2 \mathrm{~h}$. Following this treatment, sections were incubated with the EnVison complex at $37^{\circ} \mathrm{C}$ for additional $30 \mathrm{~min}$ before incubation with substrate solution 3,3'-diaminobenzidine (DAB; Beyotime Institute of Biotechnology). The sections were then counterstained with hematoxylin, and pictures taken on Olympus BH2 microscope at x200 magnification. Mean positive indices (MPI) of IHC staining were analyzed semi-quantatively by information management system (IMS) cell image analysis system, and calculated as the pixel values of positive areas $\mathrm{x}$ optical density.

Statistical analysis. Data are expressed as means \pm SD. The significance of the data was determined by Student's t-test (two-tailed) in two groups and one-way ANOVA in multiple groups. Values of $\mathrm{P}<0.05$ were considered statistically significant. All data were analyzed with SPSS 16.0 software.

\section{Results}

Lentiviruses effectively transduced MDA-MB-231 cells. Two LVs were produced: LV-IGF-1R shRNA carries shRNA targeting IGF-1R and LV-con carries only pLL3.7. All LVs expressed EGFP which allowed for titering in HeLa cells as well as measuring their infection efficiency in MDA-MB-231 cells. To permit high-efficiency transduction, we cultured the 
A

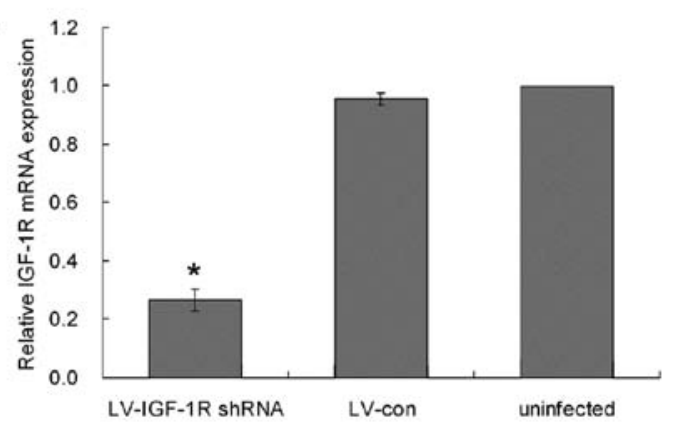

B

LV-IGF-1R shRNA LV-con uninfected

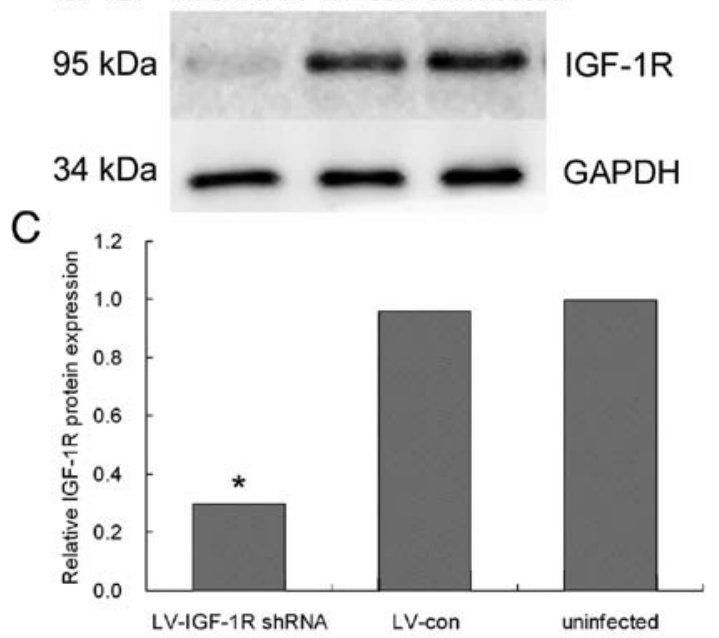

Figure 2. Suppression of IGF-1R expression in MDA-MB-231 cells by LV-IGF-1R shRNA. (A) IGF-1R mRNA expression was measured by realtime PCR using total RNA isolated from the cells. (B) Western blot analysis of IGF-1R protein expression using total protein extracted from the cells. (C) Quantification of three independent experiments from (B). ${ }^{*} \mathrm{P}<0.01$.

cells in the presence of IGF-1 and subjected them to two more rounds of lentiviral transduction with concentrated vector on 3 consecutive days. The transfection rate was calculated by the percentage of fluorescent cells in the total cells and was almost $100 \%$ in each visual field (Fig. 1). When the transduced cells were maintained in vitro for 30 days in the presence of growth factors, $>90 \%$ of the cells continued to express EGFP.

Effect of LV-IGF-IR shRNA on IGF-IR expression. To evaluate silencing efficiency, infected cells were characterized for IGF-1R mRNA by quantitative reverse transcriptionPCR (qRT-PCR) using specific primers against endogenous IGF-1R; and for protein expression in immunoblots obtained using the rabbit polyclonal antibody against IGF-1R. As shown in Fig. 1B, qRT-PCR results indicate that endogenous IGF-1R mRNA expression was significantly inhibited at $24 \mathrm{~h}$ after infection in MDA-MB-231 cells. Compared with the control group, the IGF-1R shRNA group showed lower quantities of IGF-1R mRNA; mRNA expression was decreased by nearly $70 \%$ (Fig. 2A). In accordance with this, western blot analysis showed that IGF-1R protein expression was significantly suppressed in the IGF-1R shRNA group compared with the control LV group in MDA-MB-231 cells $(\mathrm{P}<0.01)$ (Fig. 2B). IGF-1R expression was unaltered in cells infected by vectors lacking the shRNA cassette.

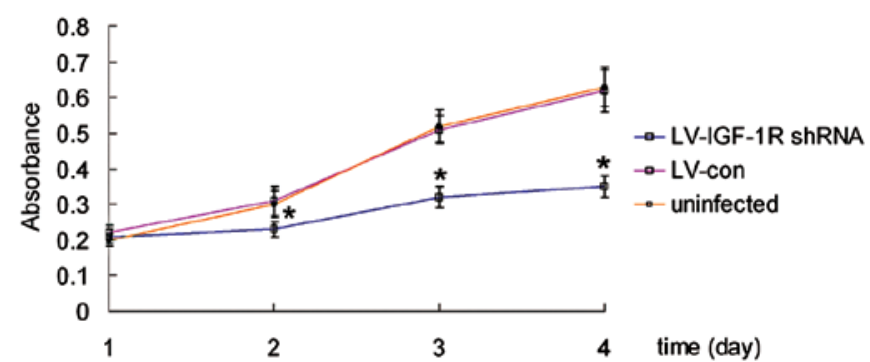

Figure 3. CCK-8 assay of MDA-MB-231 cells infected with different LVs. LV-IGF-1R shRNA significantly inhibited breast cancer cell growth in vitro. ${ }^{*} \mathrm{P}<0.01$.

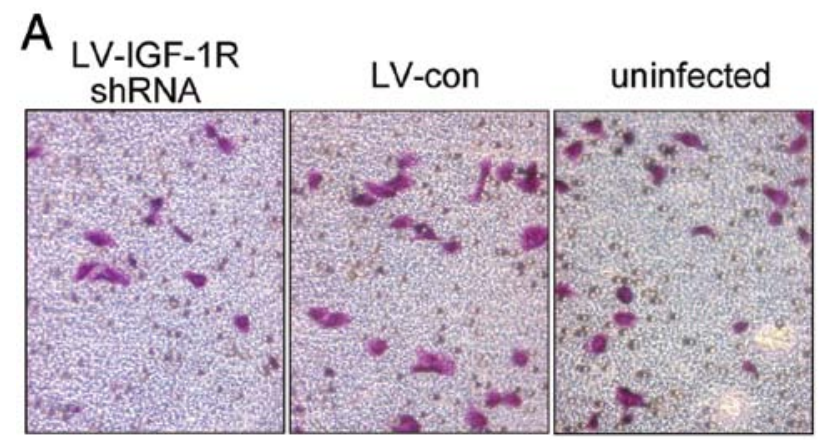

B

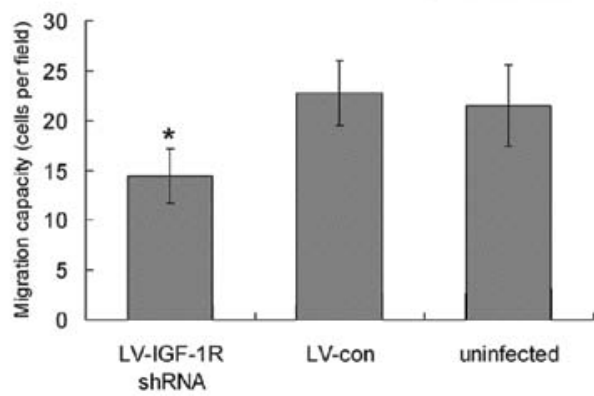

Figure 4. Migration capability examination of infected MDA-MB-231 cells by Transwell migration assay. (A) Cells stained purple by crystal violet were counted under light microscopy at x200 magnification. LV-IGF-1R shRNA significantly inhibited breast cancer cell migration in vitro. (B) Quantification of three independent experiments from (A). ${ }^{*} \mathrm{P}<0.01$.

LV-IGF-IR shRNA inhibits breast cancer cell growth in vitro. To detect effects of suppression of IGF-IR expression on cell proliferation, we carried out a CCK-8 assay using equal number of cells infected with LV-IGF-1R shRNA or LV-con. Results indicate that transfection with LV-IGF-1R shRNA inhibited MDA-MB-231 cell proliferation by 37.3 and $43.5 \%$ compared with the control group at 48 and $72 \mathrm{~h}$, respectively $(\mathrm{P}<0.01)$ (Fig. 3).

LV-IGF-IR shRNA inhibits breast cancer cell migration in vitro. We next assessed effects of IGF-1R silencing on cell motility using Transwell migration assays. Transfection of MDA-MB-231 cells with anti-IGF-1R shRNA inhibited cell migration through the polycarbonate membrane by $36.3 \%$, whereas the control shRNA had no effect (Fig. 4).

Transfection of LV-IGF-IR shRNA inhibits MDA-MB-231 cell growth in vivo. To investigate the effects of IGF-1R 


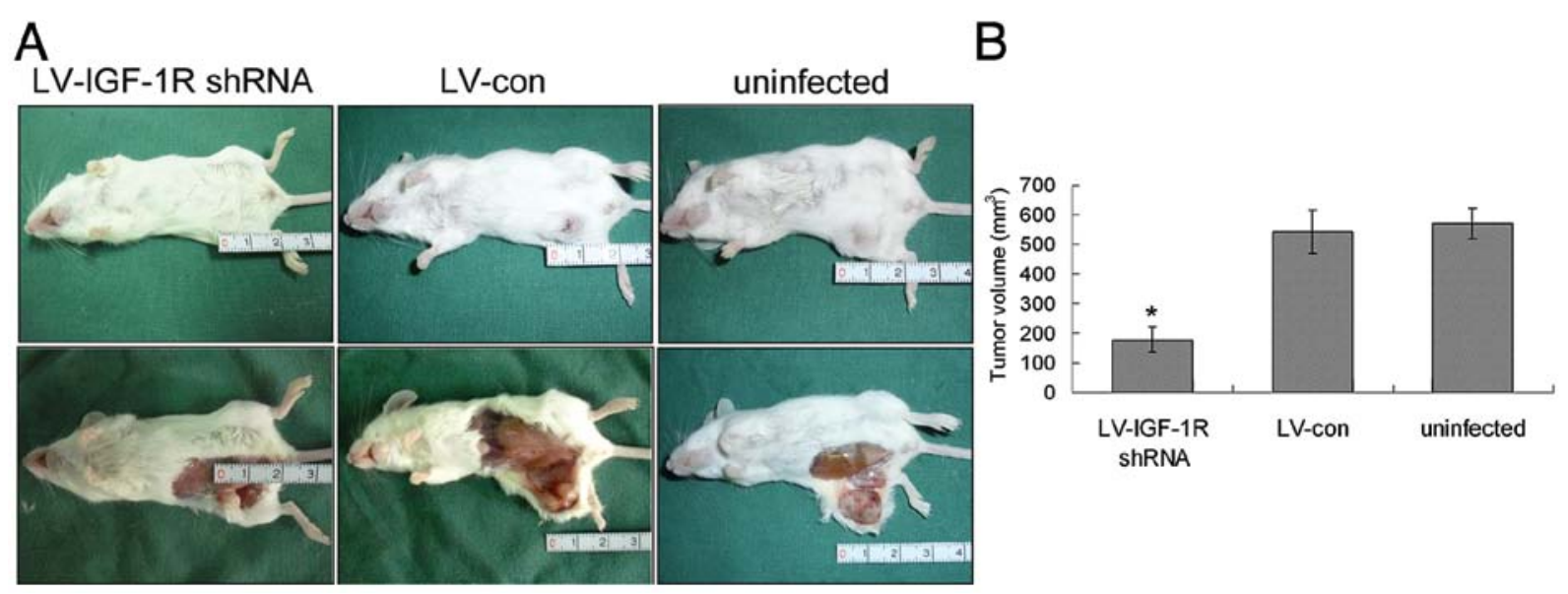

Figure 5. Effects of LV-IGF-1R shRNA on the growth of MDA-MB-231 cells xenografted into mice. (A) The SCID mice were sacrificed 2 months after transplantation and the tumors were measured. Compared with the LV-con group and the uninfected group, tumor growth in SCID mice of the LV-IGF-1R shRNA group was significantly inhibited. (B) Quantification of tumor volume in each group from (A). "P<0.01.

A

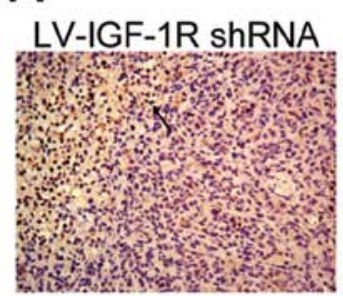

C

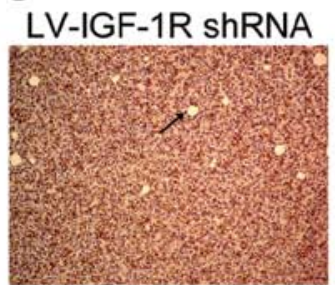

LV-con

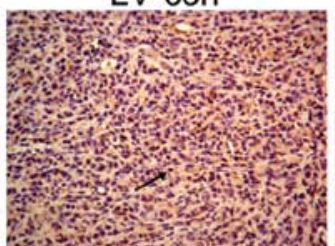

LV-con

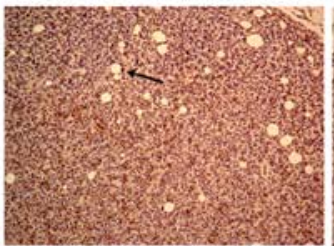

uninfected

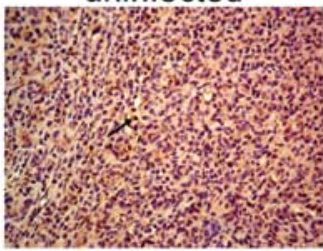

uninfected

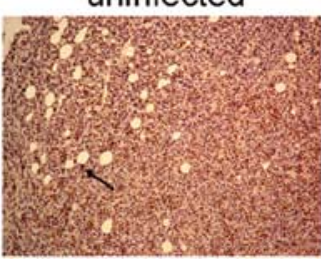

B

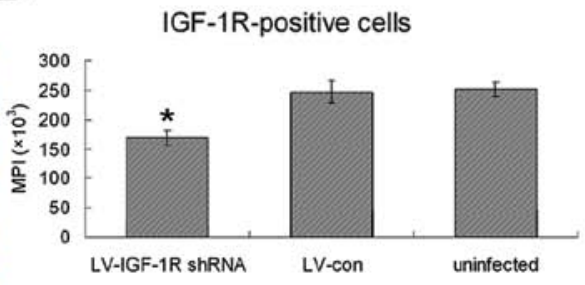

D

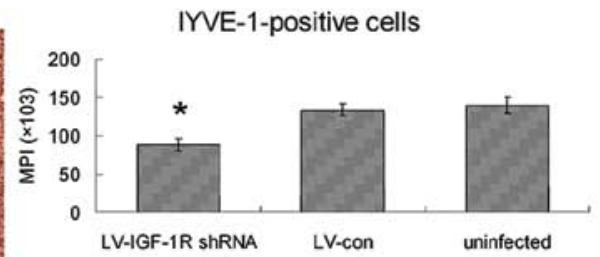

Figure 6. Suppression of IGF-1R expression and tumor lymphangiogenesis by lentivirus-mediated IGF-1R shRNA. (A) Photomicrographs of representative $5-\mu$ m-thick formalin-fixed sections immunostained with IGF-1R obtained at x 200 magnification from LV-IGF-1R shRNA group and control tumors. Arrow marks dark brown spots that are positively stained for IGF-1R. (B) Quantitation of IGF-1R ${ }^{+}$cells in immunostained sections derived from IGF-1R-shRNA group and control tumors. IGF-1R expression is inhibited by $31.5 \%$ in LV-IGF-1R shRNA group. (C) Photomicrographs of representative sections immunostained with LYVE-1 obtained at x200 magnification. The arrow marks LYVE-1 ${ }^{+}$cells. (D) Quantitation of LYVE- $1^{+}$cells in immunostained sections of each group. The tumor lymphangiogenesis of LV-IGF-1R shRNA group was inhibited by $34.2 \%$. MPI, mean positive indices. ${ }^{*}<0.01$.

silencing on cell growth in vivo, we injected SICD mice with infected MDA-MB-231 cells as described above. There was no evidence of weight loss or physical distress resulting from the treatment protocol. As shown in Fig. 5, the tumor growth of LV-IGF-1R shRNA group was significantly inhibited (66.8\% decrease).

Effect of LV-IGF-IR shRNA on tumor lymphangiogenesis. The immunohistochemical analysis for IGF-1R showed the expression level of IGF-1R in xenograft was significantly decreased in LV-IGF-1R shRNA goup, compared with the LV-con group (Fig. 6A). The effect of LV-IGF-1R shRNA on lymphangiogenesis was determined by immunohistochemical analysis using LYVE-1 antibody. The semi-quantitative data from the IMS cell image analysis is shown in Fig. 6D. The tumor lymphangiogenesis of LV-IGF-1R shRNA group was inhibited by $34.2 \%$, compared with control tumors.

\section{Discussion}

We have demonstrated in this study that shRNA delivered by a lentivirus was able to effectively suppress targeted IGF-1R expression in breast cancer MDA-MB-231 cells leading to significant suppression of cell growth and migration both in vitro and in vivo. These results encourage further exploration of RNAi as a potential method for breast cancer treatment.

Although knocked-down IGF-1R expression in some other cancer cells has been reported by a few groups using siRNA (18-20), here we showed a stable silencing of IGF-1R in MDA-MB-231 cells with LV-mediated shRNA. Other 
approaches to abrogating IGF-IR signalling include dominant negative mutants, kinase defective mutants, anti-sense oligonucleotides, soluble receptors, antibodies against IGF-I and IGF-II, IGF-IR blocking antibodies and, more recently, a family of IGF-IR kinase inhibitors (21), but the stability and delivery efficacy of these antibodies or inhibitors seems to be a crucial limiting factor in exerting an inhibitory effect on the targeted molecule in vivo. Current data support the notion that in mammalian cells, due to the obvious amplification effect of $\mathrm{RNAi}$, it is superior to antisense approaches for downregulation of gene expression though antisense has been widely used (22). Our study has indicated that the suppressive effect of LV-mediated shRNA to IGF-IR can reach about $70 \%$.

The role of IGF-1R in regulating tumor growth is well understood (23). Several studies have shown that IGF-IR plays an important role at critical steps of the metastatic cascade, including cell adhesion, migration, invasion, angiogenesis, and cell growth at distant organ sites (24). It has been demonstrated that IGF-1R regulates metastasis of colon cancer as colon cancer cells expressing dominant negative IGF-1R failed to form liver metastases following splenic injection or direct injection into the livers of nude mice (25). Expression of antisense IGF-1R mRNA inhibited Ewing's sarcoma (ES) cell motility, and their ability to form colonies in soft agar in vitro; the metastatic ability of ES cells carrying antisense IGF-1R was significantly reduced in vivo (26). Metastatic uveal melanomas express higher levels of IGF-IRs than primary tumors (27), and are sensitive to IGF-IR targeting (28). These data suggest IGF-1R is critical to metastasis of several types of cancer cells. Our study has also demonstrated that LV-mediated shRNA targeting IGF-1R significantly decreased breast cancer cell MDA-MB-231 proliferation and migration both in vitro and in vivo, indicating an important role of IGF-1R in breast cancer growth and metastasis.

Besides facilitating migration, there are two other ways in which IGF-1R can influence the spread of breast cancer to distant sites. The first is by stimulating angiogenesis and the second is by promoting lymphangiogenesis. Although angiogenesis is important for the dissemination of many solid tumors, the major way in which breast cancer cells metastasize is through the lymphatics (29). Therefore, the processes governing lymphangiogenesis may be of central importance to this disease (30). Lymphangiogenesis is another important mechanism by which tumor cells are disseminated via the lymphatic system. VEGF-C has been identified as mediators of this process (31). Lewis lung carcinoma subline M-27 cells transfected with human IGF-IR cDNA expressed VEGF-C and acquired a lymph node metastasizing potential in vivo, implicating the role of IGF-1R in the control of lymphatic metastasis (32). Our previous study also showed that increased VEGF-C expression was closely related to lymphangiogenesis in breast cancer invasion and lymphatic metastasis (33). Since IGF-1R is widely distributed in mammalian tissues, including blood and lymphatic vessels (34), intratumoral injections or tail vein injections of lentiviral vectors targeting cancer cells may interfere with vessel formation. In our study, breast cancer cells were infected with lentivirus vectors in vitro, and then transplanted into SCID mice. The results show that downregulation of IGF-1R inhibits lymphangiogenesis and tumor metastasis in vivo. In another previous study, we reported IGF-1 significantly increased VEGF-C expression in MDA-MB-231 breast cancer cells in vitro (35). Whether IGF-1R suppression would interfere with VEGF-C secretion is in need of further experimental demonstration. IGF-1R could be an important therapeutic target to suppress breast cancer metastasis, but a package of comprehensive and complementary research is required.

Taken together, it can be concluded that RNAi is a powerful genetic tool to reduce target gene expression. Our results also indicate that LV-mediated shRNA targeting IGF-1R offers a potent therapeutic strategy to inhibit lymphatic metastasis of breast cancer.

\section{Acknowledgements}

This study was supported by the National Natural Science Foundation of China, Grant Number 30872521.

\section{References}

1. Mackay J, Jemal A, Lee NC and Parkin DM: The Cancer Atlas. American Cancer Society, Atlanta, GA, 2006.

2. Anderson BO, Yip CH, Ramsey SD, Bengoa R, Braun $\mathrm{S}$, Fitch M, Groot M, Sancho-Garnier H and Tsu VD: Breast cancer in limited-resource countries: health care systems and public policy. Breast J 12: S54-S69, 2006

3. Parkin DM, Bray F, Ferlay J and Pisani P: Global cancer statistics, 2002. CA Cancer J Clin 55: 74-108, 2005.

4. Parkin DM and Fernández LM: Use of statistics to assess the global burden of breast cancer. Breast J 12: S70-S80, 2006.

5. Sachdev D: Regulation of breast cancer metastasis by IGF signaling. J Mammary Gland Biol Neoplasia 13: 431-441, 2008.

6. Achen MG and Stacker SA: Tumor lymphangiogenesis and metastatic spread - new players begin to emerge. Int J Cancer 119: 1755-1760, 2006

7. Ward CW, Garrett TP, McKern NM, Lou M, Cosgrove LJ, Sparrow LG, Frenkel MJ, Hoyne PA, Elleman TC, Adams TE, et al: The three dimensional structure of the type I insulin-like grow th factor receptor. Mol Pathol 54: 125-132, 2001.

8. Baserga R, Peruzzi F and Reiss K: The IGF-1 receptor in cancer biology. Int J Cancer 107: 873-877, 2003.

9. Shimizu C, Hasegawa T, Tani Y, Takahashi F, Takeuchi M, Watanabe T, Ando M, Katsumata N and Fujiwara Y: Expression of insulin-like growth factor 1 receptor in primary breast cancer: immunohistochemical analysis. Hum Pathol 35: 1537-1542, 2004.

10. Taunk NK, Goyal S, Moran MS, Yang Q, Parikh R and Haffty BG: Prognostic significance of IGF-1R expression in patients treated with breast-conserving surgery and radiation therapy. Radiother Oncol 96: 204-208, 2010.

11. Casa AJ, Dearth RK, Litzenburger BC, Lee AV and Cui X: The type I insulin-like growth factor receptor pathway: a key player in cancer therapeutic resistance. Front Biosci 13: 3273-3287, 2008.

12. Takeshita F and Ochiya T: Therapeutic potential of RNA interference against cancer. Cancer Sci 97: 689-696, 2006.

13. Brummelkamp TR, Bernards R and Agami R: A system for stable expression of short interfering RNAs in mammalian cells. Science 296: 550-553, 2002.

14. Brummelkamp TR, Bernards R and Agami R: Stable suppression of tumorigenicity by virus-mediated RNA interference. Cancer Cell 2: 243-247, 2002.

15. Elbashir SM, Harborth J, Lendeckel W, Yalcin A, Weber K and Tuschl T: Duplexes of 21-nucleotide RNAs mediate RNA interference in cultured mammalian cells. Nature 411: 494-498, 2001.

16. Rubinson DA, Dillon CP, Kwiatkowski AV, Sievers C, Yang L, Kopinja J, Rooney DL, Zhang M, Ihrig MM, McManus MT, et al: A lentivirus-based system to functionally silence genes in primary mammalian cells, stem cells and transgenic mice by RNA interference. Nat Genet 33: 401-406, 2003.

17. Addgene, Inc.: http://www.addgene.org/11795/. Accessed March 15,2012 . 
18. Fang J, Zhou Q, Shi XL and Jiang BH: Luteolin inhibits insulinlike growth factor 1 receptor signaling in prostate cancer cells. Carcinogenesis 28: 713-723, 2007.

19. Patel BB, Gupta D, Elliott AA, Sengupta V, Yu Y and Majumdar AP: Curcumin targets FOLFOX-surviving colon cancer cells via inhibition of EGFRs and IGF-1R. Anticancer Res 30: 319-325, 2010.

20. Santen RJ, Fan P, Zhang Z, Bao Y, Song RX and Yue W: Estrogen signals via an extra-nuclear pathway involving IGF-1R and EGFR in tamoxifen-sensitive and -resistant breast cancer cells. Steroids 74: 586-594, 2009.

21. Werner $\mathrm{H}$ and Bruchim I: The insulin-like growth factor-I receptor as an oncogene. Arch Physiol Biochem 115: 58-71, 2009.

22. Nagy P, Arndt-Jovin DJ and Jovin TM: Small interfering RNAs suppress the expression of endogenous and GFP-fused epidermal growth factor receptor (erbB1) and induce apoptosis in erbB1overexpressing cells. Exp Cell Res 285: 39-49, 2003.

23. Lann D and LeRoith D: The role of endocrine insulin-like growth factor-I and insulin in breast cancer. J Mammary Gland Biol Neoplasia 13: 371-379, 2008.

24. Samani AA, Yakar S, LeRoith D and Brodt P: The role of the IGF system in cancer growth and metastasis: overview and recent insights. Endocr Rev 28: 20-47, 2007.

25. Reinmuth N, Fan F, Liu W, Parikh AA, Stoeltzing O, Jung YD Bucana CD, Radinsky R, Gallick GE and Ellis LM: Impact of insulin-like growth factor receptor-I function on angiogenesis, growth, and metastasis of colon cancer. Lab Invest 82: 1377-1389, 2002.

26. Scotlandi K, Maini C, Manara MC, Benini S, Serra M Cerisano V, Strammiello R, Baldini N, Lollini PL, Nanni P, et al: Effectiveness of insulin-like growth factor I receptor antisense strategy against Ewing's sarcoma cells. Cancer Gene Ther 9: 296-307, 2002
27. Mallikarjuna K, Pushparaj V, Biswas J and Krishnakumar S: Expression of insulin-like growth factor receptor (IGF-1R), c-Fos, and c-Jun in uveal melanoma: an immunohistochemical study. Curr Eye Res 31: 875-883, 2006.

28. Girnita A, All-Ericsson C, Economou MA, Aström K, Axelson M, Seregard S, Larsson O and Girnita L: The insulin-like growth factor-I receptor inhibitor picropodophyllin causes tumor regression and attenuates mechanisms involved in invasion of uveal melanoma cells. Clin Cancer Res 12: 1383-1391, 2006.

29. Cunnick GH, Jiang WG, Gomez KF and Mansel RE: Lymphangiogenesis and breast cancer metastasis. Histol Histopathol 17: 863-870, 2002.

30. Kucab JE and Dunn SE: Role of IGF-1R in mediating breast cancer invasion and metastasis. Breast Dis 17: 41-47, 2003.

31. Achen MG, McColl BK and Stacker SA: Focus on lymphangiogenesis in tumor metastasis. Cancer Cell 7: 121-127, 2005.

32. Tang Y, Zhang D, Fallavollita L and Brodt P: Vascular endothelial growth factor $\mathrm{C}$ expression and lymph node metastasis are regulated by the type I insulin-like growth factor receptor. Cancer Res 63: 1166-1171, 2003.

33. Gu Y, Qi X and Guo S: Lymphangiogenesis induced by VEGF-C and VEGF-D promotes metastasis and a poor outcome in breast carcinoma: a retrospective study of 61 cases. Clin Exp Metastasis 25: 717-725, 2008

34. Björndahl M, Cao R, Nissen LJ, Clasper S, Johnson LA, Xue Y, Zhou Z, Jackson D, Hansen AJ and Cao Y: Insulin-like growth factors 1 and 2 induce lymphangiogenesis in vivo. Proc Natl Acad Sci USA 102: 15593-15598, 2005.

35. Zhu C, Qi X, Chen Y, Sun B, Dai Y and Gu Y: PI3K/Akt and MAPK/ERK1/2 signaling pathways are involved in IGF-1induced VEGF-C upregulation in breast cancer. J Cancer Res Clin Oncol 137: 1587-1594, 2011. 